\title{
ASCENDING EXCITATORY AND INHIBITORY MOTOR ACTIVITY OF COLONIC LONGITUDINAL AND CIRCULAR MUSCLES IN RAT MODEL
}

\author{
Zornitsa V. Gorcheva, \\ Galya Ts. Stavreva1, \\ Negrin N. Negrev ${ }^{2}$, \\ Radomir G. Radomirov ${ }^{3}$ \\ Department of Nursing Care, \\ Medical University - Pleven, \\ Bulgaria \\ ${ }^{1}$ Department of Pharmacology and \\ Toxicology, \\ Medical University - Pleven, \\ Bulgaria \\ ${ }^{2}$ Medical University "Prof. Paraskev \\ Stoyanov" - Varna, \\ Bulgaria \\ 'Institute of Neurobiology, \\ Bulgarian Academy of Sciences, \\ Sofia, \\ Bulgaria
}

\section{Summary}

In this experiment we studied the role of excitatory and inhibitory neurotransmissions in the ascending reflex pathways in isolated rat colon. Partitioned organ bath, electrical field stimulation (EFS), drugs and isolated preparations were used to evaluate motor activity of (LM) and circular muscles (CM). Ascending motor responses of $\mathrm{LM}$ and $\mathrm{CM}$ were frequency-dependent contraction, significantly more expressed in LM. Atropine $(0.3 \mu \mathrm{M})$ decreased ascending contractions of LM. During atropine treatment spantide $(0.1 \mu \mathrm{M})$ further suppressed ascending contractile motor responses. In the presence of atropine, L-NNA $(0.5 \mathrm{mM})$ restored ascending contractions of LM, while contractions were strongly depressed after addition of L-arginine $(0.5 \mathrm{mM})$. Ascending response in $\mathrm{CM}$, caused by atropine, consisted of an initial relaxation followed by contraction. Spantide decreased the contraction. L-NNA reduced the relaxation and significantly restored the atropineinfluenced contraction, while L-arginine induced a deep relaxation of CM. The presence of ChAT, SP-containing nerve cell bodies and fibers and NADPH-diaphorase-reactive cell bodies and processes in myenteric ganglia were detected. The results indicated that nitric oxide is an important modulator of ascending cholinergic and tachykininergic excitation in colonic region of the large intestine of rats.

Key words: ascending reflex, rat colon, atropine, L-arginine, L-NNA, spantide

\section{Introduction}

Motility of the colon is an object of experimental and clinical studies because of the medical and social significance of neutrally mediated diseases of the large intestine. The motor activity of the colon occurs in isolated preparations, indicating that the nerve structures that that induce the processes of movement are within the wall of the intestine [1-5]. The intrinsic pacemaker mechanisms determining the generation and spread of motor complexes of the colon are preserved in in vitro conditions, despite the fact that the transit along the colon in vivo could be impaired [6]. The colonic rhythmic contractions observed in mammalian experimental models seem to be equivalent to the high amplitude propagating contractions, characterizing the human large intestines [7]. 
The specific role of longitudinal muscle (LM) or circular muscle (CM) in the coordinated colonic motor activity is a matter of debate. In rat colon, the LM contracted and relaxed in a fashion reverse to that of the CM during the peristaltic reflex [8]. The polarized neural reflex in the murine large intestine could be triggered by longitudinal stretch [9], while the ascending excitatory and descending inhibitory neural pathways in guinea pig distal colon depend on sensory elements within the CM layer [10]. In rat colon, an essential role in ascending reflex contractility was attributed to LM [3]. Obviously, although that knowledge the colon motility has advanced during the last years, the autonomic regulation of colonic motility and the mechanisms underlying ascending and descending neuromuscular communications are still not fully understood $[11,12]$ and require further elucidation.

In this study, we examined neurally-mediated colonic motility, using segment preparations isolated from rat colon. The motor activity was pharmacologically tested with cholinergic-, tachykininergic- and nitrergic-related drugs to explicate the role of excitatory and inhibitory neurotransmissions in the ascending reflex pathways.

\section{Materials and Methods}

\section{Animals and preparations}

The study was approved by the Bulgarian Food Safety Agency (Protocol № 36/18.06.2015). The experiments were performed in compliance with the national (Ordinance No 15, February 03, 2006) and international laws and polices (EEC Council Directive 86/609, December 12, 1987).

The experiment was carried out on 24 male Wistar rats (body weight 272.4 $\pm 23.5 \mathrm{~g}$ ), purchased from the Research and Laboratory Animal Breeding, Slivnitsa (Bulgaria). The animals were kept at a temperature of $22 \pm 2^{\circ} \mathrm{C}$ and humidity of $50 \pm 10 \%$. They were fed with normal pelleted diet and water ad libitum was given. The rats were fasted overnight, stunned by a blow on the neck and decapitated. The abdominal cavity was opened and the distal part of the large intestine was removed. A 55-60 mm long section of colon with intact nerve plexuses and smooth muscle layers integrity was isolated
[13] and mounted horizontally in partitioned organ bath.

\section{Experimental equipment}

Partitioned organ bath for studying the peristaltic reflexes in isolated intestinal preparations was used [14]. The spontaneous or electricallyinduced motor activity of LM and CM belonging to the proximal part of the colonic segment were synchronously registered with strain gauges after preloading of preparations in longitudinal and circular axis equivalent to $10 \mathrm{mN}$.

Electric field stimulation (EFS) with the following characteristics: $0.8 \mathrm{~ms}, 40 \mathrm{~V}, 2$ or 5 or $10 \mathrm{~Hz}, 20 \mathrm{~s}$ was applied by two platinum electrodes $(0.45 \mathrm{~mm}$ thick $)$, placed diametrically opposite each other and $14 \mathrm{~mm}$ apart along the sides of organ baths at an interval of at least 5 $\min [14,15]$.

The mechanographic on-line recording techniques were completed by strain gauges and amplifiers (Microtechna, Prague, Czech Republic), stimulators (Experimetria, Budapest, Hungary) and recorders TZ 4620 (Laboratorni pristroje, Prague, Czech Republic).

\section{Protocol of the experiment}

The colonic preparations were allowed to equilibrate for a period of $30 \mathrm{~min}$ before starting the registration of the spontaneous activity and electrically-induced responses.

Application of EFS to the oral compartment of the organ bath elicited 'local' motor responses of LM and CM of the proximal part of the colonic segment, provoked by excitation of local nerve networks lying in the site of stimulation [13]. The EFS applied in the anal compartment of the bath induced 'ascending' motor responses of both muscle layers in the proximal part of the preparation at a distance of $20 \mathrm{~mm}$ away from the site of application of EFS, due to propagation of excitation via orally directed reflex pathways supplying LM and CM. The pattern and amplitude of motor responses to $5 \mathrm{~Hz}-$ stimulation resembled the pattern and amplitude of the spontaneous contractions of rat colon [3, 13] and were considered to be appropriate for evaluation of drug effects.

\section{Solutions and drugs for motor activity studies}


The modified Krebs solution $(\mathrm{NaCl} 120 \mathrm{mM}$, $\mathrm{NaHCO}_{3} 15.4 \mathrm{mM}, \mathrm{KCl} 5.9 \mathrm{mM}, \mathrm{CaCl}_{2} 2.5 \mathrm{mM}$, $\mathrm{NaH}_{2} \mathrm{PO}_{4} 1.2 \mathrm{mM}, \mathrm{MgCl}_{2} 1.2 \mathrm{mM}$, and glucose $11.5 \mathrm{mM}$ ), continuously aerated by $95 \% \mathrm{O}_{2}$ and $5 \% \mathrm{CO}_{2}(\mathrm{pH} 7.2)$ at $36.5^{\circ} \mathrm{C}$ was used.

The following substances were used: atropine sulfate (Merck, Germany), [D-Arg ${ }^{1}$, D-Trp ${ }^{7,9}$, Leu ${ }^{11}$-Substance P (Spantide), L-arginine (L-arg) and NG-nitro-L-arginine (L-NNA) (Sigma Chemicals, USA), tetrodotoxin (TTX, Sankyo, Switzerland). The stock solutions were prepared with distilled water and diluted to their final concentration in the Krebs solution before treatment. The stock solutions of TTX and Spantide were stored at $-20^{\circ} \mathrm{C}$. The drugs were added in volumes not exceeding $0.5-1.0 \%$ of the volume of compartment. The concentration and the contact time of drugs were as follows: TTX $(0.1 \mu \mathrm{M}, 10 \mathrm{~min})$, atropine $(0.3 \mu \mathrm{M}, 15 \mathrm{~min})$, Spantide $(0.1 \mu \mathrm{M}, 15 \mathrm{~min}), \mathrm{NG}$-nitro-L-arginine (0.5 mM, $15 \mathrm{~min})$ and L-arginine $(0.5 \mathrm{mM}, 15$ min). When the drugs were added consecutively (atropine plus Spantide or atropine plus NGnitro-L-arginine or atropine plus L-arginine) the time course of drug action was $30 \mathrm{~min}$.

\section{Immunohistochemical histochemical assessment}

and

The presence of substance $\mathrm{P}$ in myenteric plexus of the colonic segment was tested by immunohistochemistry according to $\mathrm{Hsu}$ et al (1981) [16], and the presence of nitric oxide by histochemistry for nicotinamide adenine dinucleotide phosphate (NADPH)-diaphorase according to Sherer-Singler et al. (1983) [17].

\section{Statistical analysis}

As a baseline for measuring the amplitude (in $\mathrm{mN}$ ) of motor events, we accepted the lowest point of the amplitudes of spontaneous highamplitude contractions. Data were processed statistically using one-way ANOVA, followed by post hoc LSD multiple comparison test and presented as mean values \pm S.E.M. We assumed that the differences between studied groups were statistically significant if $\mathrm{p}$-value $<0.05$.

\section{Results}

\section{Spontaneous motor activity}

The spontaneous motor activity of the LM and $\mathrm{CM}$ were presented as irregular high-amplitude contractions as seen in the longitudinal or circular axis of the colonic segment preparations. The tissue tone did not change considerably over a period of about 150-180 min. The contractions of both muscles could be considered as moving in anal direction complex with a frequency $0.52 \pm 0.07$ cycles per $\min (n=24)$. The amplitudes of the contractions of the LM, $10.8 \pm 1.9 \mathrm{mN}$ $(n=24)$ were higher than those of the CM, $5.1 \pm 0.8 \mathrm{mN}(\mathrm{n}=24, \mathrm{p}<0.05)$ - Figure $1 \mathrm{~A})$. The contractions of the LM and CM occurred synchronously, with a delay in appearance of the CM contractions (Figure 1B).

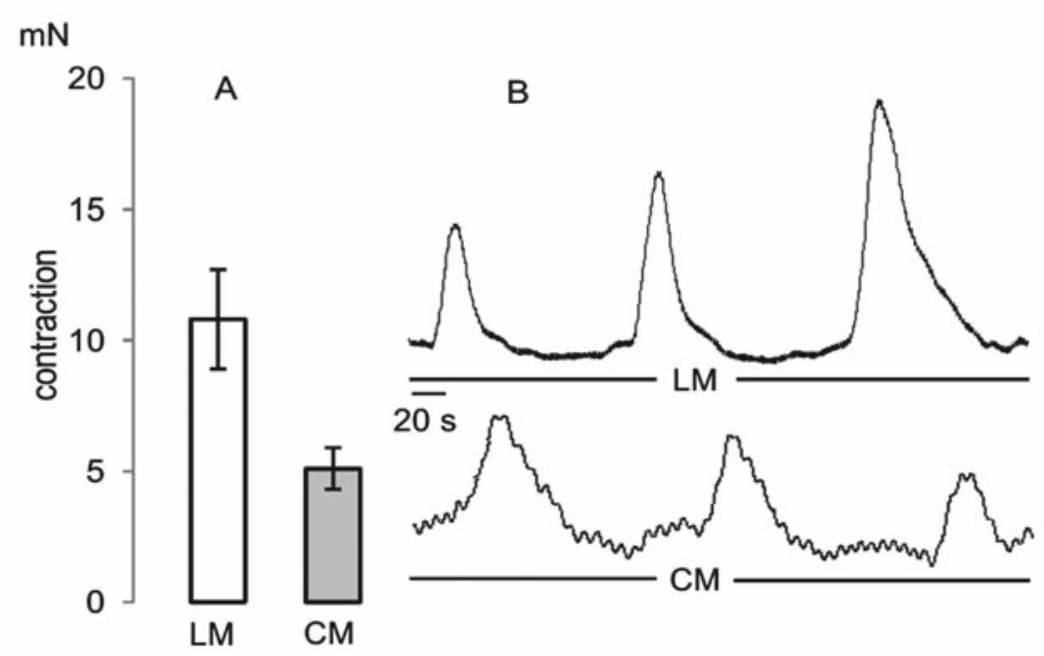

Figure 1. Typical mechanographic records showing spontaneous motor activity of smooth muscles in proximal part of isolated rat colonic segments (B) and the amplitude of contractions (A). Designations: longitudinal (LM) and circular (CM) muscles 


\section{Electrically-induced local motor responses}

Local motor responses of both LM and CM were elicited by applying electrical stimulation to the proximal part of the colonic segments.

The local responses of LM were frequency- dependent contractions, and the contractions induced by $2-\mathrm{Hz}$ electrical stimulation were significantly less pronounced compared to those provoked by $5-\mathrm{Hz}$ or $10-\mathrm{Hz}$ stimulation (Figure $2 \mathrm{~A}, \mathrm{~B}, \mathrm{C})$.

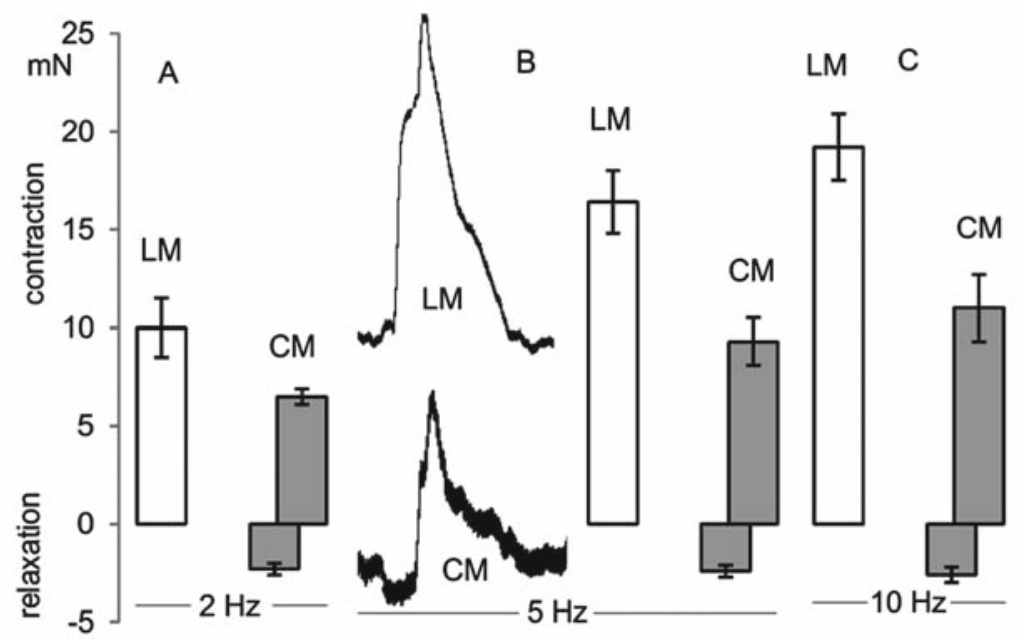

Figure 2. Local motor responses of isolated rat colonic segments to electric stimulation applied at frequencies of $2 \mathrm{~Hz}(\mathrm{~A}), 5 \mathrm{~Hz}(\mathrm{~B})$ and $10 \mathrm{~Hz}(\mathrm{C})$. Designation: longitudinal (LM) and circular (CM) muscles

The responses of CM consisted of an initial relaxation followed by a contraction (Figure 2 ; B). The amplitudes of relaxation in the local responses of CM were not dependent on the frequency of EFS, while the contractions became markedly stronger with the increase of the frequency of the electrical stimuli (Figure 2;

\section{Electrically-induced motor responses}

The ascending motor responses of LM and CM of the proximal part of the preparations were frequency-dependent contractions (Figure $3 \mathrm{~A}$, $\mathrm{B}, \mathrm{C})$. $\mathrm{A}, \mathrm{B}, \mathrm{C})$.
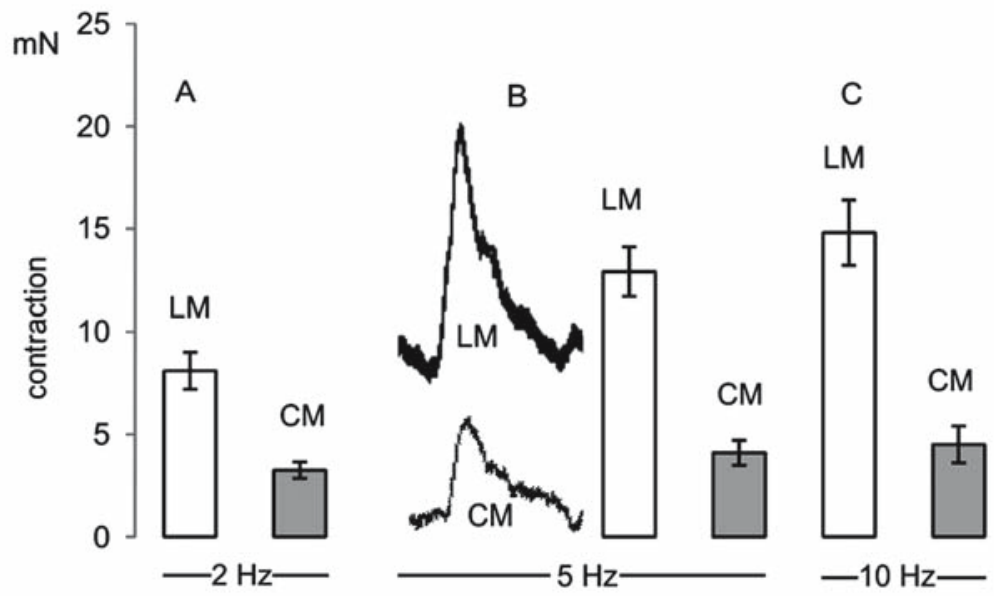

Figure 3. Ascending motor responses of isolated rat colonic segments to electric stimulation applied at frequencies of $2 \mathrm{~Hz}(\mathrm{~A}), 5 \mathrm{~Hz}(\mathrm{~B})$ and $10 \mathrm{~Hz}(\mathrm{C})$. Designation: longitudinal (LM) and circular (CM) muscles

The amplitudes of the ascending contractile responses of LM were significantly higher comparing to the amplitudes of the ascending responses of $\mathrm{CM}$ induced by all the frequencies of electrical stimulation used (at $5 \mathrm{~Hz}$-stimulation, $\mathrm{LM}, 12.9 \pm 1.2 \mathrm{mN}$ and $\mathrm{CM}, 4.1 \pm 0.6 \mathrm{mN}, \mathrm{n}=12$, 
$\mathrm{p}<0.05)$ - Figure 3; B.

The amplitudes of the ascending contractions were considerably less expressed, as compared to the local contractile responses in both LM and CM.

\section{Drug effects on the electrically- elicited ascending motor responses}

Atropine $(0.3 \mu \mathrm{M})$ decreased the ascending contractions of LM elicited by EFS, applied at a frequency of $5 \mathrm{~Hz}(5.40 \pm 0.60 \mathrm{mN}, \mathrm{n}=10$, $\mathrm{p}<0.05$ vs. control). The addition of NK1 antagonist spantide $(0.1 \mu \mathrm{M})$ on the background of atropine further suppressed the amplitudes of the ascending contractile motor responses $(3.2 \pm 0.3 \mathrm{mN}, \mathrm{n}=10, \mathrm{p}<0.05)$. In the presence of atropine, L-NNA $(0.5 \mathrm{mM})$ restored to a great extent the ascending contractions of LM, while the amplitudes of contractions were strongly decreased after adding L-Arg $(0.5 \mathrm{mM})$ - Figure 4.

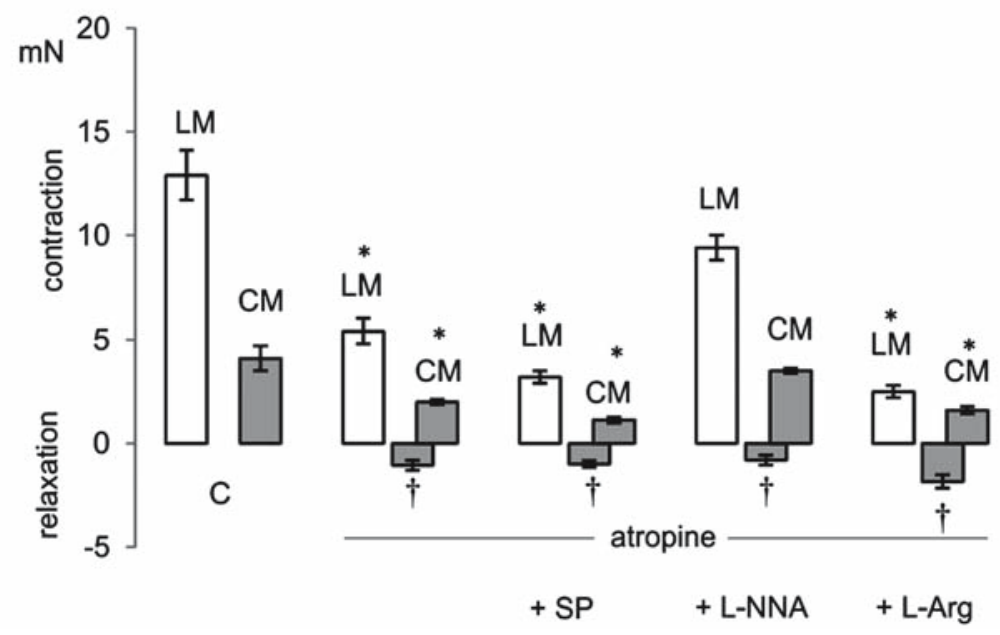

Figure 4. Ascending motor responses of longitudinal and circular muscles in isolated rat colonic segments to electric stimulation applied at frequencies of $5 \mathrm{~Hz}$. Designation: control (C) and in the presence of atropine, atropine plus spantide (Sp), atropine plus L-NNA, atropine plus L-arginine. The values represent means \pm S.E.M. of at least eight experiments. Designation: longitudinal (LM), circular (CM) muscles; significant differences at $\mathrm{p}<0.05-(*)$ vs. controls, $(\dagger)$ opposite effects vs. controls.

In the presence of atropine $(0.3 \mu \mathrm{M})$, the ascending responses in $\mathrm{CM}$ of proximal part of the colonic preparation induced by electrical stimulation of $5 \mathrm{~Hz}$ converted from contractions to responses, consisting of a relaxation $(1.05 \pm 0.12 \mathrm{mN}, \mathrm{n}=12)$ followed by contraction ( $2.0 \pm 0.24 \mathrm{mN}, \mathrm{n}=12, \mathrm{p}<0.05$ vs. ascending contractile responses). Spantide $(0.3 \mu \mathrm{M})$, added on the background of atropine decreased the contraction to $1.12 \pm 0.16 \mathrm{mN}(\mathrm{n}=8)$ without changing the relaxation. L-NNA $(0.5 \mathrm{mM})$, a competitive inhibitor of nitric oxide synthase slightly reduced the amplitude of relaxation and restored the atropine-influenced contraction $(3.5 \pm 0.24 \mathrm{mN}, \mathrm{n}=8, \mathrm{p}<0.05)$. L-Arg $(0.5 \mathrm{mM})$ deepened the relaxation in atropine-pretreated colonic preparations (Figure 4).

\section{Effect of TTX}

The addition of TTX $(0.1 \mu \mathrm{M})$ for $10 \mathrm{~min}$ to oral or anal part of colon segments prevented the local and ascending motor responses of LM and $\mathrm{CM}$, respectively.

\section{Immunohistochemical and histoche- mical testing}

The immunohistochemical studies showed the presence of SP-immunostained cell bodies and nerve fibers, which ran between the myenteric ganglia and the muscle layers (Figure $5 \mathrm{~A}, \mathrm{~B}$ ).

NADPH-diaphorase-reactive multipolar nerve cell bodies and their processes outlined myenteric ganglia. NADPH-diaphorase-positive neurons and numerous varicose fibers in the ganglia and internodal strands were seen (Figure 5 C, D). 

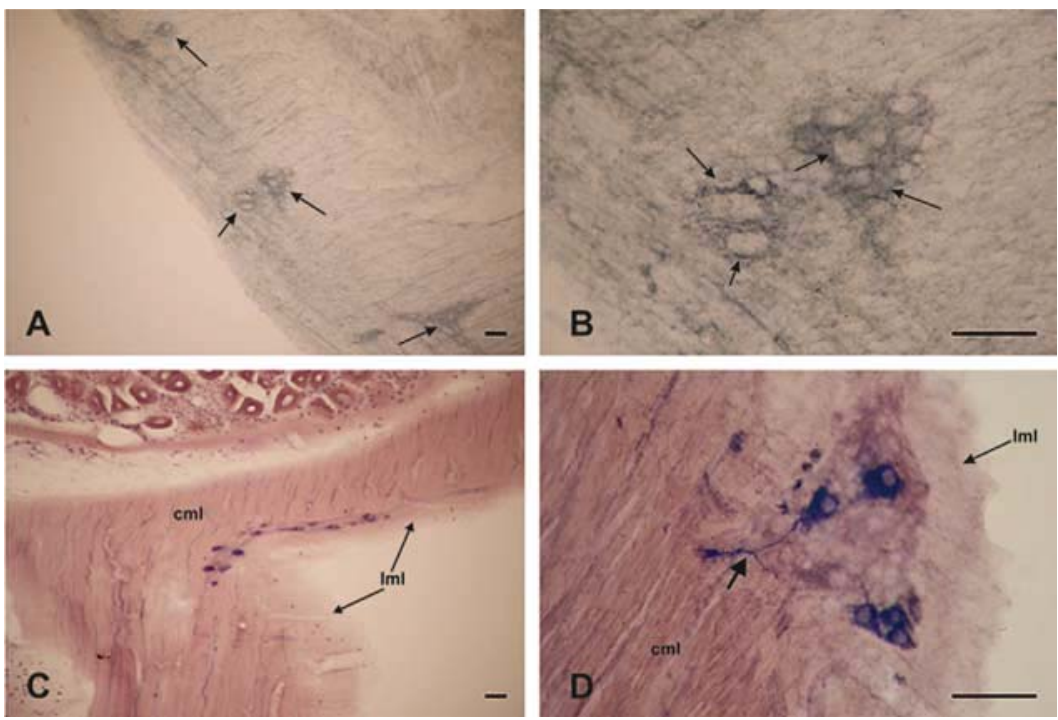

Figure 5. Photomicrographs illustrating substance P immunohistochemistry (A, B) and NADPH-d histochemistry $(\mathrm{C}, \mathrm{D})$ in proximal part of colonic preparation. A - SP-immunoreactive nerve fibers outlined myenteric ganglia (arrows) in proximal part of the colon. B - Detail from A. SP-immunonegative neurons are outlined (arrows) by immunopositive varicose nerve fibers. $\mathrm{C}-\mathrm{NADPH}$-diaphorase-positive nerve cell bodies in the myenteric ganglions and nerve fibers in the internodal strands. D - Myenteric ganglion containing NADPH-d-positive and negative neurons in the proximal part of colon. Large arrow points to varicose and branching positive axon in the myenteric ganglion. Scale bar $=50 \mu \mathrm{m}$

\section{Discussion}

This study evaluated motor responses in gut preparations isolated from rat colon. According to Dalziel et al. (2014) [18] segments of intact rat large intestine, and hence intact enteric neural circuitry, provide an ideal in vitro model for investigation of colonic motility. The partitioned organ bath used in this study allowed simultaneous recording of the motility of LM and $\mathrm{CM}$ of the segment-preparations as a display of enteric nerve-dependent functional coordination in the motor activity of both muscles.

The spontaneous high-amplitude contractions of LM and CM in isolated segments of rat proximal colon we established are in concert with the described high-amplitude contractions in rat proximal, middle and distal colon [3, 14, 19, 20]. The rhythmic high-amplitude contractions observed in the large intestine of many mammals seem to be equivalent to the propagating contractions in humans [7]. We found that the contractions of LM were significantly higher compared to contractions of $\mathrm{CM}$ and they appeared synchronously in both muscles layers. The fact that contractile activity was recorded in the proximal part of the segments suggests the propagation of the contractile events from the proximal to the distal part of the rat colon. On the contrary, in the guinea pig, the distal colon is the most likely region to induce a peristaltic wave, if compared with the mid- or proximal colon [21]. Relaxations were not registered in the spontaneous motor activity of LM and CM that could be due to the lack of fecal material and filling the lumen of the segments with Krebs solution in such experimental conditions.

The application of EFS to the oral or to the distal part of the preparations elicited local or ascending motor responses of LM and CM belonging to the proximal part of the colonic segments. The effect of electrical stimulation resulted from the action of excitatory and/or inhibitory neurotransmitters, which are released as a consequence of propagation of action potential along the nerve terminals [15, 22]. The motor responses of rat colonic LM and CM induced by EFS were neurogenic by nature, since they were prevented by tetrodotoxin, an inhibitor of firing the action potential in neurons. TTX blocked the motor patterns in isolated segments of the guinea-pig proximal and distal colon [5].

The electrically-induced responses, as well as spontaneous high-amplitude contractions appeared synchronously in LM and CM, 
suggesting co-activation of neural circuits supplying both muscles. The increase of stimulus frequency enhanced the amplitudes of local and ascending responses without changing the motor pattern, thus suggesting that the increase of the responses corresponded to the frequencydependent release of the same neurotransmitters. It is known that more nerve terminals are involved in the process of the neurotransmitter release when electrical stimuli are applied at higher frequencies [23].

Our experiments showed that in the rat proximal colon the local motor responses of both muscles, which were considered to result from the local excitation of the modular nerve structures [24] were different. Comparison with the other tubular smooth muscle organs shows that a wide variety of motor patterns occurs in the large intestine. This variety suggests a relatively autonomic nervous system in the gut wall [25]. Although the stimulatory effects of pelvic nerve stimulation and inhibitory effects of inferior mesenteric ganglia could not be excluded from the regulation of colonic motility (guineapig distal colon [11], the neural mechanisms underlying the colonic motor patterns have now been identified to lie within the myenteric plexus and do not require inputs from the mucosa or submucosal ganglia for their generation [25]. We found that under local electrical stimulation the LM responded with contraction, while the response of CM consisted of an initial relaxation followed by contraction. The relaxation could be due to the prevalence of inhibitory innervation serving the $\mathrm{CM}$ of the rat colon. The neurallyevoked release of the inhibitory neurotransmitter nitric oxide in human colon is greater in $\mathrm{CM}$ than in LM [26].

We noticed that the electrically-activated ascending motor responses of LM and CM in rat colonic segments manifested as contractions. Similarly to both high-amplitude contractions observed in the spontaneous motor activity and contractile components of the local motor responses, the ascending contractions of $\mathrm{CM}$ were significantly less expressed as compared to the contractions of LM, most probably demonstrating a predominant role of the LM in the colonic contractile coordination mechanisms. More recently, ascending responses, different in pattern and resulting from nerve excitation, were reported in murine distal colon, in which nerve stimulation produced both inhibition and excitation on CM and LM [12].

The ascending electrically-induced contractions of LM and CM were decreased by atropine, a blocker of muscarinic receptors and spantide, a tachykinin receptor antagonist thus demonstrating that excitatory cholinergic and tachykininergic neurotransmissions are involved in the ascending motor reflex pathways supplying the rat proximal colon. In the presence of atropine, a relaxation in the ascending contractile responses of CM revealed. L-NNA, an inhibitor of nitric oxide synthase decreased while L-arginine, a substrate of nitric oxide synthesis increased the ascending relaxations of CM. On the background of atropine the ascending contractile responses of LM were increased by L-NNA and were decreased by L-arginine suggesting that nitrergic neurotransmission plays role in ascending responses of both muscles of rat colon. The persistence of relaxing response in the presence of L-NNA confirms the existence and participation of other inhibitory neurotransmitters, in addition to nitric oxide effect on motor responses of CM [12]. Relaxation was not observed in the ascending LM responses when the ascending excitatory cholinergic and tachykininergic neurotransmissions were inhibited. This is most likely due to ascending nitric oxide-dependent reflex pathways, whose function is not so well expressed in LM as compared to $\mathrm{CM}$ in rat colon.

Cholinergic and tachykininergic neurotransmissions have been widely recognized as playing a key role in neural circuits, serving the motor events of the large intestine of several mammalians, including colonic region of mouse [27] and guinea pig [28]. More recent observations have demonstrated that opioidergic [18] and GABAergic [29] neurotransmissions could modulate the motility of the colon, too. We found that nitric oxide-related drugs affected the ascending motor responses of the colonic LM and CM. Despite the assumption that nitric oxide is a principal neurotransmitter in descending inhibitory motor pathways [30, 31 ], our results indicated that nitric oxide is an important modulator of ascending cholinergic and tachykininergic excitation in colonic region of the large intestine of rats. 


\section{Conclusions}

The partitioned organ bath used in this study allowed registration of excitatory and inhibitory activity of local as well as ascending reflex motor responses of LM and CM in the proximal part of colonic region in a rat experimental model. Cholinergic and tachykininergic neuronal and neuromuscular communications provided contractile responses of both muscles. A dominant contractility of LM was observed, suggesting an essential role in coordinated contractile processes. Nitrergic mechanisms are involved in modulation of ascending cholinergic and tachykininergic excitation but nitric oxidedependent reflex pathways supply rather $\mathrm{CM}$ than LM. The presence of Substance P- and NADPHdiaphorase-positive nerve structures proved the physiological significance of substance $\mathrm{P}$ and nitric oxide in nerve pathways in the rat colonic region.

\section{Acknowledgments}

This study was supported by grants from the Medical University - Pleven, Bulgaria and Medical University - Varna, Bulgaria.

\section{References}

1. Grubišića V, Verkhratskyc A, Zorece R, Parpura V. Enteric glia regulate gut motility in health and disease. Brain Res Bull. 2018;136:109-17.

2. Nicholas S, Spencer NJ. Peristalsis and fecal pellet propulsion do not require nicotinic, purinergic, 5-HT3, or NK3 receptors in isolated guinea pig distal colon. Am J Physiol Gastrointest Liver Physiol. 2010;298(6):G95261.

3. Nedialkova N, Stavreva G, Negrev N, Ivancheva C, Radomirov R. Functional coordination of motor activity in colonic smooth muscles in rat experimental model. Physiol Res. 2011;60(4):659-66.

4. Kendig DM, Hurst NR, Bradley ZL, Mahavadi $\mathrm{S}$, Kuemmerle JF, Lyail V, et al. Activation of the umami taste receptor (T1R1/T1R3) initiates the peristaltic reflex and pellet propulsion in the distal colon. Am J Physiol Gastrointest Liver Physiol. 2014;307(11):1100-7.

5. Costa M, Wiklendt L, Simpson P, Spencer NJ, Brookes SJ, Dinning PG. Neurochemical factors involved in the formation and propulsion of fecal pellets in the guinea pig colon. Neurogastroenterol Motil. 2015;27(10):146677.

6. Spencer NJ, Kyloh M, Wattchow DA, Thomas A, Sia TC, Brookes SJ, et al. Characterization of motor patterns in isolated human colon: are there differences in patients with slow-transit constipation? Am J Physiol Gastrointest Liver Physiol. 2011;302(1):G34-43.

7. Smith TK, Park KJ, Henning GW. Colonic migrating motor complexes, high amplitude contractions, neural reflexes and the importance of neuronal and mucosal serotonin. $\mathrm{J}$ Neurogastroenterol Motil. 2014;20(4):423-46.

8. Grider JR. Reciprocal activity of longitudinal and circular muscle during intestinal peristaltic reflex. Am J Physiol Gastrointest Liver Physiol. 2003;284(5):G768-75.

9. Heredia DJ, Dickson EJ, Bayguinov PO, Henning GW, Smith TK. Colonic elongation inhibits pellet propulsion and migrating motor complexes in the murine large bowel. J Physiol. 2010 ;588(Pt 15):2919-34.

10. Spencer NJ, Dickson EJ, Henning GW, Smith TK. Sensory elements within the circular muscle are essential for mechanotransduction of ongoing peristaltic reflex activity in guinea-pig distal colon. J Physiol. 2006;576(Pt 2):519-31.

11. Gribovskaja-Rupp, Babygirija R, Takahashi $\mathrm{T}$, Ludwig K. Autonomic nerve regulation of colonic peristalsis in Guinea pigs. J Neurogastrienterol Motil. 2014;20(2):185-96.

12. Zhang Y, Paterson WG. Characterization of the peristaltic reflex in murine distal colon. Can J Physiol Pharmacol. 2016;94(2): 190-8.

13. Brading AF, Ivancheva C, Radomirov $\mathrm{R}$. Functional coordination of motor activity in colonic and recto-anal smooth muscles in rat experimental model. Meth Find Exp Clin Pharmacol. 2008;30(3):201-7.

14. Ivancheva $\mathrm{C}$, Radomirov R. Excitatory ascending and descending motor responses in the guinea pig small intestine: a comparative study of longitudinal and circular muscles by a triple bath method. Meth Find Exp Clin Pharmacol. 2001;23(5):223-9.

15. Paton WD, Vizi ES. The inhibitory action of noradrenaline and adrenaline on acetylcholine output by guinea-pig ileum longitudinal muscle strip. Br J Pharmacol. 1969;35(1):10-28.

16. Hsu SM, Raine L, Fanger H. Use of avidinbiotin-peroxidase complex (ABC) in immunoperoxidase techniques: a comparison between $\mathrm{ABC}$ and unlabeled antibody (PAP) procedure. J Histochem Cytochem. 1981;29(4):577-80.

17. Sherer-Singler U, Vincent SR, Kimura $H$ 
McGeer EG. Demonstration of a unique population of neurons with NADPHdiaphorase histochemistry. J Neurosci Methods. 1983;9(3):229-34.

18. Dalziel JE, Spencer NJ, Dunstan KE, Lynch AT, Haggarty NW, Gopal PK, et al. An in vitro rat model of colonic motility to determine the effect of $\beta$-casomorphin-5 on propagating contractions. Food Funct. 2014;5(11):2768-74.

19. Gonzalez A, Sarna SK. Different types of contractions in rat colon and their modulation by oxidative stress. Am J Physiol Gastrointest Liver Physiol. 2001;280(4):G546-54.

20. Mitsui R, Ono S, Karaki S, Kuwahara A. Propionate modulates spontaneous contractions via enteric nerves and prostaglandin release in rat distal colon. Jon J Physiol. 2005;55(6):3318.

21. Kwak JM, Babygirija R, Gribovskaja-Rupp I, Takahashi T, Yamato S, Ludwig K. Regional difference in colonic motility response to electrical field stimulation in Guinea pig. J Neurogastroenterol Motil. 2013;19(2):192-203.

22. Kadlec O, Schurkes J, Seferna I. The topographical basis and frequency-dependence in the effect of different compounds on neurogenic contractions of the guinea pig ileum. Gen Physiol Biophys. 1993;12(1):69-83.

23. Kadlec O, Seferna I, Sevcik G, Somogyi GT, Vizi ES. The topographical basis of cholinergic transmission in guinea pig ileum myenteric plexus. Neuroscience 1990;36(3):793-802.

24. Costa M, Brookes SJ, Henning GW. Anatomy and physiology of the enteric nervous system. Gut. 2000;47(Suppl 4):iv15-9.
25. Spencer NJ, Dunning PG, Brookes SJ, Costa M. Insights into the mechanisms underlying colonic motor patterns. J Physiol. 2016;594(1):4099116.

26. McKirdy HC, Richardson CE, Green JT, Rhodes J, Williams GT, Marshall RW. Differential effect of nitric oxide synthase inhibition on sigmoid colon longitudinal and circular muscle responses to nicotine and nerve stimulation in vitro. Br J Surg. 2004;91(2):229-34.

27. Brierley SM, Nichols K, Grasby DJ, Waterman SA. Neural mechanisms underlying migrating motor complex formation in mouse isolated colon. Br J Pharmacol. 2001;132(2):507-17.

28. Bian XC, Heffer LF, Gwynne RM, Bornstein JC, Bertrand PP. Synaptic transmission in simply motility reflex pathways excited by distension in guinea pig distal colon. Am J Physiol Gastrointest Liver Physiol. 2004;287(5):G101727.

29. Auteri M, Zizzo MG, Mastropaolo M, Serio R. Opposite role played by GABAA and GABAB receptors in the modulation of peristaltic activity in mouse distal colon. Eur J Pharmacol. 2014;731:93-9.

30. Schneider S, Wright CM, Heuckeroth RO. Unexpected roles for the second brain: enteric nervous system as master regulator of bowel function. Annu Rev Physiol. 2019;81:235-59.

31. Radomirov R, Ivancheva C, Itzev D, Petkova-Kirova P. Locality-dependent descending reflex motor activity in the anal canal-cholinergic and nitrergic contributions in the rat model. Acta Pharmacol Sin. 2009;30(9):1276-82. 